\title{
Ausländerbehörden im dynamischen Feld der Migrationssteuerung ${ }^{1}$
}

Tobias G. EULE

\section{Einleitung}

Dieses Kapitel beleuchtet die Rolle von Ausländerbehörden, den Behörden, die regulär über den Verbleib von Migrant*innen zu entscheiden haben, im Kontext ihres Verhältnisses zu anderen staatlichen und nichtstaatlichen Akteuren. Der vorliegende Text baut dabei auf bisheriger ethnographischer Forschung zur Rechtsanwendung in Ausländerbehörden auf (vgl. Eule 2014). Die wenigen bestehenden Studien in diesem Feld gehen in der Regel von einem kooperativen und mehr oder weniger guten Verhältnis zwischen staatlichen Kontrollakteuren aus und weisen zivilgesellschaftlichen Akteuren eine kritisch betrachtende oder aktiv intervenierende Rolle zu (vgl. Ellermann 2009; Cyrus und Vogel 2003; Feldman 2012). Dieses recht einfache Bild soll auf Basis von 20 Monaten ethnographischer Feldforschung an fünf Standorten korrigiert werden. Konkret zeigt das Kapitel, dass Beziehungen zwischen staatlichen Akteuren sehr wohl auch konfliktgeladen sind, während Beziehungen zwischen Ausländerbehörden und zivilgesellschaftlichen Organisationen gerade in der Verwaltungskrise 2015 kooperativ und unterstützend waren. Zivilgesellschaftliche Akteure sind daher nicht mehr grundsätzlich als kritische Überwacher, sondern als wohlwollende und in Einzelfällen kritische Begleiter der Arbeit von Ausländerbehörden zu ver-

1 Die Datenerhebung für diesen Beitrag wurde durch Forschungsförderung des Economic and Social Research Council UK (Ref. ES/M003825/1) und des Schweizer Nationalfonds (Ref. 100017_153225) ermöglicht. 
stehen. Diese inzwischen viel dynamischeren Beziehungen sind auf drei Entwicklungen zurückzuführen, die am Ende des Beitrags beleuchtet werden sollen.

Das Kapitel beruht sowohl auf den Ergebnissen der ursprünglichen Feldforschung, als auch auf aktuellen Tätigkeiten des Autors im Rahmen von Beratungstätigkeiten und neuen Feldaufenthalten im Sommer 2015. Neben teilnehmenden Beobachtungen wurden dabei über 70 Interviews mit Experten aus dem Umfeld von Ausländerbehörden geführt - mit Vertretern anderer Ämter, Gerichte, und vielen unterschiedlichen zivilgesellschaftlichen Organisationen. Da der vorliegende Text auf ethnographischer Feldforschung beruht, stellen Vignetten aufbereitete Feldnotizen - einen großen Teil der hier genutzten Daten dar. Aus Platzgründen wird dabei darauf verzichtet, die einzelnen Feldkontexte besonders einzuführen; die vorgestellten Ausschnitte sollen der Argumentation dienen.

Der nächste Abschnitt dient als kurze Einführung in die Aufgaben und $\mathrm{Zu}$ ständigkeiten von Ausländerbehörden sowie den bisherigen Forschungsstand. Danach werden die »kollegialen « Behörden beschrieben, denen in der Regel ein konstant gutes Verhältnis zu Ausländerbehörden zugeschrieben wird: andere Ausländerbehörden, Sozialämter, Polizei und das Bundesamt für Migration und Flüchtlinge (BAMF). Der Schwerpunkt des Kapitels liegt auf der Beschreibung des Verhältnisses von Ausländerbehörden und Institutionen, denen die Literatur ein eher kritisches Verhältnis zuspricht, vornehmlich Akteuren der Zivilgesellschaft. Deren in Wirklichkeit komplexe und teilweise unterstützende Verbindung zu Ausländerbehörden wird in einem dritten Teil beschrieben, bevor schließlich drei Ursachen benannt werden, die einen Wandel im Feld der Migrationsverwaltung hervorgebracht haben: Veränderungen von Recht, der Ausrichtung und Zuständigkeit von Akteuren.

\section{Was sind Ausländerbehörden?}

Während im konkreten Asylverfahren die Prüfung über die Plausibilität und Glaubwürdigkeit eines einzelnen Antrags Spezialisten mit zumindest einer gewissen zentralisierten Ausbildung obliegt, werden wie in vielen anderen europäischen Ländern alle anderen aufenthaltsrechtlichen Belange von kommunalen Ausländerbehörden behandelt. Dies umfasst im Asylkontext sowohl die Ausstellung und Verlängerung der Gestattung des Aufenthalts zur Durchführung des Asylverfahrens (nach §55 Abs. 1 AsylVfG), die Ausstellung und Verlängerung von Aufenthaltserlaubnissen »aus völkerrechtlichen, humanitären oder politischen Gründen« (nach Kapitel 2, Abschnitt 5 des Aufenthaltsgesetzes), als auch die Ausweisung und Durchführung aufenthaltsbeendender Maßnahmen (nach 
dem fünften Kapitel des Aufenthaltsgesetzes) sowie in den meisten Fällen die Entscheidung über eine mögliche Einbürgerung der Betroffenen. Während die Mitarbeitenden des Bundesamts für Migration und Flüchtlinge also im Asylprozess an sich eine erhebliche Entscheidungsmacht ausüben, sind alle daraus resultierenden Aufenthaltstitel und die Frage nach dem möglichen Weiterverbleib oder der Ausreise Aufgabe der Ausländerbehörden.

Da Ausländerbehörden im Gegensatz zu den Außenstellen des BAMF in Trägerschaft der Kreise und kreisfreien Städte liegen, unterscheiden sich erstere ganz erheblich in Größe, Ausstattung und Professionalisierung. Während in kleinen, ländlichen Kreisen mit geringem Migrant*innenanteil oft Einzelpersonen »die« Ausländerbehörde darstellen, haben Ausländerbehörden in Hamburg oder Berlin durchaus mehrere 100 Mitarbeitende. Auch wenn sie die im Großen und Ganzen gleiche Rechtsgrundlage (das Aufenthaltsgesetz und zugehörige Verfahrensvorgaben sowie zahlreiche andere nationale und europäische Rechtsquellen) haben, können der Arbeitsalltag und die Anwendungspraxis in Ausländerbehörden äußerst unterschiedlich sein. Hierzu gibt es bisher relativ wenig wissenschaftliche Daten (vgl. Boswell und Geddes 2011; Feldman 2012; Morris 2002). Eine von mir durchgeführte ethnographische Untersuchung der Arbeitspraxis von vier unterschiedlichen Behörden (Eule 2014) bestätigt aber, dass gerade in der Entscheidungsfindung und Sachverhaltsklärung sowohl innerhalb als auch zwischen Ausländerbehörden immense Unterschiede bestehen. Diese Unterschiedlichkeit scheint dabei mit der zunehmenden Prekarität des Aufenthaltsstatus zu steigen: je unsicherer der Verbleib in Deutschland, desto unterschiedlicher die Verwaltungspraxen in den beforschten Ämtern.

Ausländerbehörden sind damit also nicht nur wirkungsmächtige Akteure in der Verwaltung von Asyl, sondern auch in vielen Fällen unberechenbar. Dies liegt an vielen Faktoren, etwa der hohen Komplexität und ständigen Veränderung des geltenden Rechts oder der relativ geringen Vergütung und schlechten Ausbildung der Mitarbeitenden. In keiner der vier Behörden gab es zum Zeitpunkt der Feldforschung ein strukturiertes Einarbeitungs- oder Fortbildungskonzept, so dass neue Sachbearbeiter*innen einerseits ihrer Eigeninitiative überlassen waren, andererseits aber durch das Zuschauen und Abgucken etablierter behördlicher Praktiken lernten. Im Ergebnis lag in allen beforschten Ämtern ein hoher Grad an Informalität vor, der zwar einerseits Mitarbeitenden Hilfe und Leitung anbot, andererseits aber Rechtsanwendung stärker als erwartet (oder manchem lieb sein mag) von mündlichen Überlieferungen und nicht Textkenntnis abhängig machte. Sachbearbeiter*innen entschieden dabei nicht gänzlich willkürlich, sondern in eigener (und oftmals kollektiver) Interpretation und Anwendung tradierter Geschichten über das Recht (vgl. Eule 2014). Neben internen 
Faktoren spielen dabei auch diverse externe Faktoren eine große Rolle, auf die in diesem Beitrag der Schwerpunkt gelegt werden soll.

Das Gros der Forschungsliteratur zu Migration hat bisher über die Frage der praktischen Migrationsverwaltung einen weiten Bogen gemacht (vgl. Boswell und Geddes 2011; Morris 2002). Mit der Abwendung vom Nachzeichnen breiter nationaler (und stereotyper) Unterschiede zwischen Migrationspolitiken und der Hinwendung zu kleinteiligeren, weniger normativen und komplexeren Steuerungsstrategien (vgl. Geiger und Pécoud 2012; Geiger und Pécoud 2010) in den letzten zehn Jahren sind auch einige Studien über Migrationsverwaltung $\gg$ in action« erschienen (z.B. Ellermann 2009; Cyrus und Vogel 2003; Eule 2014; CôtéBoucher, Infantino und Salter 2014; eine frühe und sehr mikrosoziologische Ausnahme ist Scheffer 1995; Scheffer 2001). Diese Studien unterscheiden sich in Fokus und Qualität, kommen aber oft zu dem Ergebnis, dass zwischen Migrationsverwaltung und Zivilgesellschaft ein kritisches, grundsätzlich eher antagonistisches Verhältnis besteht (v.a. Ellermann 2009; Borgards 2006; Anderson 2011). Studien wie Ellermans belegen, dass lokale zivile Akteure in der Regel gegen Ausländerbehörden mobilisieren, selbst wenn die öffentliche Meinung im Staat für schärfere Migrationskontrollen ist. Diese Annahme findet sich auch in eher theoretischen oder normativen Texten (Castles 2004a, 2004b; Hollifield 2004) und in Literatur, die sich auf den lokalen Kontext von Migration bezieht (Buckel 2011).

In der Tat findet sich diese Perspektive der kritischen zivilen Akteure auch in der Praxis wieder. Größere zivilgesellschaftliche Organisationen wie Pro Asyl agieren als wichtiges und in Teilen wirkmächtiges Kontrollorgan zu rechtspolitischen Debatten auf nationaler, aber auch lokaler Ebene. Die zwei großen Fachzeitschriften des Ausländerrechts, der Informationsbrief Ausländerrecht und die Zeitschrift für Ausländerrecht und Ausländerpolitik mit den Anwaltsnachrichten Ausländer- und Asylrecht zeigen regelmäßig auch »worst practice«-Beispiele der Migrationsverwaltung auf. Auf konzeptioneller wie textueller Ebene scheint ein grundsätzlich kritisches Verhältnis zwischen Ausländerbehörden und Zivilgesellschaft also plausibel.

Dies deckt sich in Teilen mit den Erfahrungen zur praktischen Kooperation, die im Laufe der Datenerhebung erhoben werden konnten. Aus allen Perspektiven gibt es in den Interviews Anekdoten über das historisch schlechte Verhältnis der beteiligten Akteure, über »Presseschlachten«, direkte Konfrontationen und ein grundsätzlich misstrauisches Miteinander. Gleichzeitig hatten diese Berichte immer auch einen historischen Kontext - sie stellten Erlebnisse der Vergangenheit und nicht die aktuellen Erfahrungen dar und wurden in der Regel verwendet, um auf ein inzwischen anderes Verhältnis hinzuweisen. Diesem gilt es im vor- 
liegenden Beitrag nachzuspüren. Es soll dabei aufgezeigt werden, dass verschiedene Akteure der Migrationssteuerung zwar sehr wohl auf Grund ihrer Aufgabe zusammenhängen (vgl. Feldman 2012), aber nicht notwendigerweise besonders gute Verhältnisse miteinander haben müssen. Im Gegensatz dazu ist das Miteinander zwischen Ausländerbehörden und lokalen zivilgesellschaftlichen Organisationen häufig viel weniger von Kritik als von Kooperation und gegenseitiger Unterstützung gezeichnet.

\section{Akteure der Migrationssteuerung}

Neben der Ausländerbehörde sind fünf weitere staatliche Organisationen mit der Verwaltung von Migration beschäftigt. Einerseits liegt es im Aufgabenbereich der Polizeien der Länder und des Bundes, unerlaubte Einreise, Aufenthalt und Arbeitsaufnahme zu ermitteln. Dies ist Teil des regulären Streifendienstes, es gibt aber auch in vielen Bundesländern spezielle Einheiten, in denen Daten zu diesen Straftatbeständen erfasst und ausgearbeitet werden. Darüber hinaus ist die Polizei bei verschiedenen ausländerbehördlichen Aktivitäten, gerade etwa begleiteten Abschiebungen, in Amtshilfe aktiv. Gerichte spielen in Widerspruchsfällen und bei Haftanträgen wichtige Rollen und haben darüber hinaus auch in klassisch kodifizierten »civil law«-Systemen oft eine unmittelbar rechtssetzende Funktion. Auf sie wird in anderen Kapiteln dieses Sammelbandes genauer eingegangen, weswegen sie hier weniger betrachtet werden sollen. Das Bundesamt für Migration und Flüchtlinge (BAMF) entscheidet über Asylfälle, ist aber auch in Fragen der sprachlichen Integration (über die Sprach- und Integrationskurse) sowie teilweise bei der Koordination von »freiwilliger Rückkehr« mit Rückkehrhilfen der Internationalen Organisation für Migration (IOM) aktiv. Verschiedene lokale Behörden spielen in der Verwaltung des Alltags eine große Rolle. Hierzu gehören einerseits die Arbeitsämter, die über die Anerkennung von Qualifikationen, die Einreihung in Erwerbsprogramme sowie die Beschäftigung in zustimmungspflichtigen Berufen zuständig sind. Eine zweite, noch unmittelbar wichtigere Behörde ist das Sozialamt, das in der Regel eine spezielle Stelle für Asylfragen - oft Asylbewerberleistungsstelle - hat. Hier werden Unterkunft und Versorgung der Asylbewerber*innen und anerkannten Flüchtlinge organisiert. Schließlich gibt es in manchen Bundesländern Stellen des Landes (Landesausländerbehörde, zentrale Ausländerbehörde, Landesamt für Migration oder Landesamt für Flüchtlingsfragen genannt und jeweils den Abteilungen für Ausländerrecht des jeweiligen Ministeriums für Inneres zugeordnet), die einerseits Erstaufnahmeunterkünfte für neue Asylbewerber*innen unterhalten, andererseits 
in Amtshilfe Aufgaben im Rückführungsbereich - vor allem Passbeschaffung und Massentransporte - übernehmen.

All diesen Stellen ist gemein, dass sie staatlich finanziert, beauftragt und von Beamten oder Angestellten des Staats besetzt sind. Als Behörden des Bundes, der Länder oder der Kommunen haben sie natürlich unterschiedliche Dienstherren und sind mit sehr unterschiedlich spezialisiertem und geschultem Personal besetzt, dennoch ist die staatliche Anstellung und damit Repräsentanzrolle gegenüber Migrant*innen ein entscheidender gemeinsamer Faktor. So werden Vertreter*innen von Ausländerbehörden, die bei anderen Stellen vorsprechen, sowohl im Sozialamt als auch bei der Polizei als »Kollegen« wahrgenommen dies steht im klaren Widerspruch zu den zivilgesellschaftlichen Organisationen, die im folgenden Teil besprochen werden sollen. Zum Verwaltungsgericht besteht hier eine größere Distanz, allerdings eine eindeutig größere Nähe als zwischen Gericht und einzelnen Betroffenen.

Es ist zu erwarten, dass auf Grund dieser strukturellen Nähe auch eine gewisse enge Zusammenarbeit zwischen den genannten Behörden und den Ausländerbehörden existiert. Dies stimmt in vielen Fällen auch, ist aber gleichzeitig fast immer zu qualifizieren. Nur weil zwei Behörden »den Staat« repräsentieren und oft ähnliche Aufgaben übernehmen, heißt es nicht, dass sie besonders gut kooperieren oder grundsätzlich miteinander auskommen. Gute Verhältnisse sind in der Regel nicht Ausdruck einer strukturellen Logik, sondern interpersonaler Faktoren und bedürfen eines regen und informellen Austauschs.

Bei einem Treffen regionaler Ausländerbehörden sind auch Vertreter*innen der Polizei geladen und ein Vertreter der »Fachstelle illegaler Aufenthalt und Schwarzarbeit« $\left(\right.$ FIAS) $*^{2}$ der Landespolizei ist angefragt worden, um einen Vortrag über die aufenthaltsrechtlichen Maßnahmen zu halten, die sich aus dem Schengener Visa-Kodex ergeben. Hiernach sind Visa, deren Inhaber erkennbar einem anderen Zweck nachgehen als gedacht (z.B. Touristen, die arbeiten), sofort ungültig zu erklären - die betroffene Person verliert also sofort ihre Aufenthaltsberechtigung. In der entsprechenden Region (immerhin mit ca. 3 Millionen Einwohnern) hätten Ausländerbehörden in den fünf Jahren der Geltung des Visa-Kodex bisher keinen einzigen solchen Fall bearbeitet - dies scheint den FIASVertreter ungemein zu stören. Beim gemeinsamen Mittagessen erklärt er mir seinen Frust - die Ausländerbehörden würden ihren rechtlichen Auftrag nur höchst selektiv erfüllen, dabei seien die Visa-Annullierungen ohne Ermessen auszuführen und auch noch ziemlich einfach zu machen. Die Vertreter*innen der Ausländerbehörden sehen die Sache etwas

2 Dies ist ein anonymisiertes Akronym und soll keinen Rückschluss auf das Bundesland geben. 
anders. Schon während des Vortrags wird klar, dass kaum einer der Vertreter*innen besonders aufmerksam ist, und auch bei der anschließenden Diskussion hält sich das Interesse in Grenzen. Als ich während einer Kaffeepause mit verschiedenen Teilnehmenden das Thema anspreche, winken diese nur ab. Man könne sich nicht mit jeder kleinsten EUBestimmung auseinandersetzen, das schaffe nur zusätzliche Arbeit und würde nicht helfen, Fälle abzuarbeiten. Außerdem solle doch die Polizei den Touristen hinterherschnüffeln, wenn die das so wichtig fänden.

Etwas anders ist die Lage im Spätsommer 2015, als klar wird, dass die lokale Polizei Geflüchtete nicht nur nicht an ihrer Weiterreise in Richtung Skandinavien hindert, sondern diese aktiv begleitet und etwa Familien hilft, vom Busbahnhof zum Bahnhof zu finden, ohne sie nach (natürlich nicht vorhandenen) Aufenthaltspapieren zu fragen. Eine der im Fall des Visa-Kodex noch so entspannten Ausländerbehördenvertreter*innen ist hier sehr aufgebracht und bezichtigt die Polizei der Beihilfe zum illegalen Aufenthalt - kurz, des so heiß diskutierten Menschenschmuggels. Statt Verständnis für dieses Verhalten zu zeigen, findet sie es verantwortungslos und erklärt, dass man stattdessen ja gleich das Dubliner Übereinkommen aufkünden könne.

Bei der Definition und Erfüllung ihres gesetzlichen Auftrags sind sich Polizei und Ausländerbehörde also nicht immer einig. Wie die obigen Beispiele zeigen, kann dies durchaus in unterschiedliche Richtungen gehen. Während im ersten Beispiel die Polizei die Einhaltung einer supranationalen Richtlinie für immens wichtig erklärt, ist es im zweiten Fall die Ausländerbehörde, die der Polizei selektives Verhalten und Missachten von EU-Recht vorwirft. Trotz des grundsätzlich kollegialen Verhältnisses wäre es also viel zu simpel, davon auszugehen, dass diese zwei Behörden stets am gleichen Strang ziehen und eine rechtliche oder praktische Position vertreten. Diese Meinungsverschiedenheiten sind dabei nicht trivial. Während die Zahl der mit Touristenvisum arbeitenden Personen schwer zu schätzen ist, geht man offiziell davon aus, dass 2015 mindestens 60000 Geflüchtete Deutschland gen Skandinavien wieder verlassen haben - diese Zahl beruht auf Zählungen der Polizei, die den Prozess nicht nur nicht verhindert, sondern aktiv begleitet hat.

Während das Verhältnis zur Polizei noch relativ gut ist, scheint die Beziehung zum BAMF fast traditionell schlecht. Fast alle der Ausländerbehörden, mit denen ich im Rahmen der Feldforschung in Berührung gekommen bin, beklagten sich über schlampige Arbeit, schlechte Erreichbarkeit und Unberechenbarkeit der »Kollegen« des BAMF. Dies wurde im Sommer 2015 durch die rapide ansteigenden Fallzahlen und zu spät genehmigten Personalaufstockungen noch spürbarer. In Transitstadt, meinem Feldaufenthalt 2015, wurde fast jeder zugewiesene Geflüchtete mit einer vom BAMF falsch ausgestellten Aufenthaltsge- 
stattung (dem Aufenthaltsstatus während des Asylverfahrens) zur Ausländerbehörde geschickt, was für jede betroffene Person eine zusätzliche Vorsprache und für die Mitarbeitenden der Ausländerbehörde natürlich erheblichen Mehraufwand bedeutete. Versuche, das lokale BAMF-Büro zu erreichen, scheiterten unter anderem daran, dass das Büro überhaupt nicht erreichbar war und auch auf E-Mails nicht antwortete. Als eine über das Innenministerium geleitete Anfrage mit der Aussage beantwortet wurde, man sei leider überarbeitet und könne den Fehler nicht vermeiden, schienen nicht wenige Mitarbeitende der Ausländerbehörde geneigt, mit Fackeln und Mistgabeln vor das BAMF zu ziehen. Man habe Verständnis für die schwierige Situation und sei geduldig, was Registrierungs-, Entscheidungs- oder allgemeine Kommunikationszeiten angehe. Aber mit systematischen Fehlern den ebenso überarbeiteten Ausländerbehörden zusätzliche Arbeit zu verschaffen, wurde als großer und nicht leicht heilbarer Affront wahrgenommen. Die als Retaliation geplanten Trotzreaktionen konnten zwar durch die Beschwichtigung der Behördenleitung verhindert werden, aber dennoch war im Folgenden häufiger zu beobachten, dass Anfragen vom BAMF ganz nach unten auf den Rückstandsstapel gelegt wurden.

Das Verhältnis zu Mitarbeitenden der lokalen Sozialämter war hier deutlich besser. Dies mag überraschen, da von allen genannten staatlichen Akteuren der Migrationssteuerung die Sozialämter am ehesten eine gegensätzliche Position einnehmen könnten. Solche Konflikte sind offenbar früher durchaus vorgekommen; gleichzeitig waren sich alle Ausländerbehörden darin einig, dass das Verhältnis zum Sozialamt nie so schwierig gewesen sei wie zu Akteuren der Zivilgesellschaft. Obwohl man deutlich unterschiedliche Aufgaben zu erfüllen sah, gab es aus Sicht der beteiligten Behörden keinen Grund, nicht dennoch gut miteinander auszukommen. Dies lag insbesondere daran, dass viele Mitarbeitende von Ausländerbehörden und Sozialämtern sich kannten, da sie vom selben Dienstherren (dem Landkreis oder der kreisfreien Stadt) beschäftigt wurden und sich ihre Wege so häufig gekreuzt haben. Das gute persönliche Verhältnis konnte hier die oftmals unterschiedliche rechtliche Perspektive zu Flucht und humanitärer Migration überdecken. Im Ergebnis war das Vertrauensverhältnis zu den Sozialämtern sogar stärker als das zum den Ausländerbehörden eigentlich viel näher stehenden BAMF.

In Transitstadt wurde dies dadurch besonders deutlich, dass die Asylbewerberleistungsstelle den Ausländerbehörden bei der Registrierung der zugewiesenen Asylsuchenden unter die Arme griff, in dem man die relevanten Zuweisungspapiere und den neuen Ankunftsnachweis BÜMA (ein 2015 eingeführter Status, der vorläufig anstelle einer - ebenso vorläufigen - Aufenthaltsgestattung gilt) der Neuankommenden kopierte und damit den Geflüchteten - und der Aus- 
länderbehörde - immerhin eine Vorsprache ersparen konnte. Diese Arbeitserleichterung wurde »auf kurzem Dienstweg« von zwei befreundeten Sachbearbeiter*innen organisiert, die ihre jeweiligen Vorgesetzten umgingen und vor vollendete Tatsachen stellten. Auch hier ist die persönliche und informelle Zusammenarbeit wieder symptomatisch für die Arbeit der Ausländerbehörden allgemein - viele Schwierigkeiten im Arbeitsprozess wurden durch individuelle Vorstöße geklärt.

Der obige Abschnitt zeigt also, dass die unterschiedlichen Akteure der Migrationssteuerung keinesfalls als einig oder gar koordiniert agierend verstanden werden können. Wie die Beispiele des Visumsmissbrauchs oder der irregulären Durchreise zeigen, sind Situationen, in denen Migrant*innen von laxer Rechtsdurchsetzung profitieren können, genauso Produkt von Entscheidungen spezifischer lokaler Akteure wie es etwa die Entscheidungen über die Durchführung von Abschiebungen sind. Das Verhältnis der Akteure kann durchaus von Misstrauen geprägt sein (wie häufig zwischen BAMF und Ausländerbehörden zu beobachten); ähnliches Misstrauen also, wie man zwischen Ausländerbehörden und Migrant*innen oder zivilgesellschaftlichen Akteuren erwarten könnte. Der folgende Abschnitt soll letztere Organisationen genauer ins Bild nehmen und beleuchten, in wie weit hier Kooperationen oder Friktionen vorherrschen.

\section{Akteure der Zivilgesellschaft}

Transitstadt, Frühsommer 2015. Seit Monaten steigen die Zahlen der Geflüchteten, die dringlich - untergebracht und - weniger dringlich - in den kommunalen Ausländerbehörden registriert werden müssen. Seit Monaten werden dabei den städtischen Behörden Asylbewerberleistungsstelle wie Ausländerbehörde - falsche Aufnahmezahlen aus den Erstaufnahmeeinrichtungen übermittelt, so dass erst jeden Dienstag, danach fast täglich Unterbringungen und Registrierungen improvisiert werden müssen. Um im regulären Arbeitsprozess zu bleiben, muss erst jeder Neuangekommene von der Ausländerbehörde registriert werden, um dann zu Fuß zur Asylbewerberleistungsstelle gehen. Der Weg beträgt kaum 5 Minuten, ist aber relativ schwer zu erklären, wenn man keine gemeinsame Kommunikationssprache hat. Dies jeweils individuell zu tun, kostet Zeit; Zeit, die die Mitarbeitenden der Ausländerbehörde nicht haben. Im Gegensatz zur Leistungsstelle, wo man die Zahl der Beschäftigten seit Anfang 2015 vervierfacht hat, sind hier keine zusätzlichen Stellen geschaffen worden, die Behörde ist im Gegenteil eher unterbesetzt, die Mitarbeitenden in der Situation, reguläre Termine und ungeplant im wahrsten Sinne des Wortes abgeladene Geflüchtete parallel abzuarbeiten. Von Tag zu Tag ist spürbar, wie die Sachbearbeiter*innen hektischer, nervöser, abgearbeiteter werden. Vor wie hinter den Tresen 
herrschen zunehmend chaotische Zustände - wilde Dokumentenstapel türmen sich auf und stürzen ein, Pässe gehen verloren, zu viele Menschen mit viel Gepäck drängen sich in den engen Wartebereichen, die regulären Migrant*innen beschweren sich über Wartezeiten, der Brandschutzbeauftragte über die Versperrung von Fluchtwegen.

Wie an vielen Orten in Deutschland ist das Chaos in der transitstädter Ausländerbehörde ein lösbares logistisches Problem, das durch Fehleinschätzungen und Fehlkalkulation ausgelöst wurde. BAMF und in Grenzen auch die Aufnahmestelle des Bundeslandes senden falsche Prognosen auf Grund falscher Zahlen (etwas, das erst im August aufgelöst wird), die Kommunen schätzen die Lage völlig falsch ein und begreifen erst viel zu spät, dass Geflüchtete auch Migrant*innen sind und damit auch die Ausländerbehörden zusätzlich belasten. In der Behörde selbst führen die ständige Unruhe und hohe Belastung dazu, dass bestehende, aber nicht mehr sinnvolle Prozesse nicht hinterfragt, sondern strikt befolgt werden, weil dieses immerhin gewisse Sicherheit vermittelt. Ähnlich dem von Janis beschriebenen Gruppenverhalten werden so aus eigentlich dynamischen Deliberationsprozessen sich verselbstständigende Entwicklungen, die oft idealere Lösungen ignorieren (Janis 1972). In Transitstadt befand sich die Ausländerbehörde im Sommer 2015 an einem Punkt, wo weder Vorgesetzte noch Sachbearbeitende Zeit oder Nerven für klärende Gespräche hatten und sich so Ineffizienz durchsetzte.

Der entscheidende Lösungsimpuls kam von außen. Unterstützt von verschiedenen größeren Organisationen begann ein kleiner Verein in christlicher Trägerschaft, den Fluss durch den lokalen Bürokratiewust zu organisieren. Das Gepäck der Geflüchteten wurde zwischengelagert, die Menschen in Gruppen von Behörde zu Behörde geführt, Sprachmittler*innen organisiert, um auf den Fluren zu vermitteln, erklären und beruhigen. Bemerkenswert war dabei, dass sich die Hilfsbereitschaft ausdrücklich auch auf die Behördenmitarbeitenden bezog: Die Koordinator*innen sprachen regelmäßig vor, um sich für den Einsatz der Ausländerbehörde zu bedanken, um ihr Verständnis und ihr Mitgefühl auszudrücken und zu erklären, was sie jetzt versuchten, um die Situation für alle Beteiligten zu verbessern. Neben praktischen Aspekten vor Ort gehörte dazu, dass der Verein sich mit bissigen Beschwerdemails an BAMF und Landesaufnahmestelle wandte und die Auswirkungen der chaotischen Arbeit dort anprangerte. Ein zivilgesellschaftlicher Verein, der sich bei anderen Akteuren der Migrationssteuerung für die Ausländerbehörde stark macht - eine überraschende Situation.

Der hier beschriebene Fall ist extrem, aber dennoch aussagekräftig für das veränderte Verhältnis zwischen zivilgesellschaftlichen Akteuren und der Ausländerbehörde. Das in der Literatur erwähnte klassische Beispiel - Zivilgesell- 
schaft mobilisiert gegen Ausländerbehörden - konnte in keinem der untersuchten Standorte zwischen 2009 und 2015 in Reinform gefunden werden. Stattdessen überwogen Formen der lockeren bis festen Kooperation, des Austauschs und gegenseitigen Verständnisses. Dies soll in keinem Fall heißen, dass zivilgesellschaftliche Organisationen sich notwendigerweise zu Komplizen des Aufenthaltsrechts machen, oder dass es keine unterschiedlichen Rechtsmeinungen über Einzelfälle mehr gibt. Stattdessen ist bei der Beschreibung des Verhältnisses von Ausländerbehörden und Zivilgesellschaft von einer anderen Grundfigur auszugehen. Nicht grundsätzlich kritische Überwachung und Gegenpol, sondern eine wohlwollende und in Einzelfällen kritische Begleitung beschreibt das Verhältnis in den fünf beforschten Städten.

Sowohl Ausländerbehörden als auch zivilgesellschaftliche Organisationen scheinen stark über gewachsene und überwiegend informelle Strukturen und Prozesse zu funktionieren (vgl. Eule 2014, Kapitel 4). Erfahrene Mitarbeitende als Art institutionelles Gedächtnis sind hier besonders wichtig. Ähnliches gilt mit Abstrichen - für Anwälte, wo in vielen Städten alteingesessene »Platzhirsche« den Markt beherrschen. Mit Ausnahme von einzelnen Vertreter*innen einer sehr politisch aktiven NGO, die ich zu Beginn meiner Feldforschung interviewte, waren sich aber alle Gesprächspartner*innen einig, dass sich das Verhältnis zwischen Ausländerbehörden und nichtstaatlichen Akteuren - insbesondere aber lokalen zivilgesellschaftlichen Organisationen - grundlegend geändert habe. Die bisher in der Literatur beschriebenen Eindrücke scheinen also zum Zeitpunkt der jeweiligen Erhebung durchaus plausibel gewesen zu sein (und Konflikte zwischen den Parteien gibt es ja nach wie vor), das grundsätzliche Verhältnis scheint sich aber verändert zu haben. Drei Aspekte erscheinen besonders relevant:

Vertreter*innen von Ausländerbehörden und Zivilgesellschaft stehen in einem persönlichen, informellen und vertrauensvollen Austausch. So banal es klingen mag: »wir reden jetzt direkt miteinander« wird fast durchgehend als wichtigster Faktor für die Veränderung der Beziehungen genannt. Statt schriftlich oder über Mittler (Presse, Integrationsabteilungen, Parteien) zu kommunizieren, sei es jetzt möglich, Fragen direkt miteinander zu besprechen. Zivilgesellschaftliche Organisationen scheinen nun besser über die Organisation der Ausländerbehörden informiert und in der Lage zu sein, direkte Ansprechpartner*innen zu finden, um Einzelfälle zu besprechen. Im Umkehrschluss haben Ausländerbehörden begriffen, dass ein - eventuell lästiger - direkter Austausch sinnvoller ist als ein kritischer Pressebericht. Dieses Miteinander hat in der Regel unterschiedliche Quellen. Häufig werden gemeinsame Teilnahmen an Ar- 
beitskreisen erwähnt, manchmal das Engagement einzelner »Türöffner«, manchmal schlicht und ergreifend »sich verändernde Zeiten«.

Neben der veränderten Form der Kommunikation scheint sich auch das Bild des anderen verändert zu haben. Dies zeigen die Experteninterviews. Nicht mehr »rassistische Beamtenfossile« gegen »naive Sozialromantiker« bestimmen die gegenseitigen Charakterisierungen. In der gegenseitigen Einschätzung sind die jeweiligen Organisationen professioneller und pragmatischer geworden. Dieser Eindruck deckt sich zumindest teilweise mit den erhobenen Daten aus den teilnehmenden Beobachtungen, wobei natürlich die Einschätzung hier die viel relevantere, weil Handlung anleitende Variable ist. Nicht nur kann man miteinander reden, ein Austausch kann für beide Parteien zielführend und vorteilhaft sein. Entscheidend sind hier die Erfahrungen, dass schwierige Einzelfälle so gemeinsam geklärt werden konnten. In der Folge werfen sich die beteiligten Akteure nicht mehr vor, nur von starren politischen Positionen auszugehen, sondern erkennen an, dass man in der Sache, nicht aber im Prinzip argumentiere. In den Interviews loben Vertreter*innen von großen NGOs regelmäßig die Ausländerbehörden für ihre Bereitschaft, auch mal großzügige Prognoseentscheidungen zu fällen. Andersherum betonen Mitarbeitende der Ausländerbehörden, dass zivilgesellschaftliche Akteure auch verstehen könnten, wenn rechtlich kein Spielraum gegeben sei, um einen Fall positiv zu entscheiden, und dann auch die Versagung von Aufenthalten und Ausweisungen akzeptieren würden.

Dieses andere Bild und dieser direkte Austausch finden sich in Ansätzen auch in unterschiedlichen Kooperationsstrukturen wieder. Hier sind unterschiedliche Arbeitskreise, vor allem aber einerseits Migrationsberatungen im behördlichen Alltag und andererseits sogenannte lokale Härtefallkommissionen zu betonen. Diese haben theoretisch das Potential, behördliche und zivilgesellschaftliche Ansätze miteinander zu verzahnen und verfestigen. Beratungsangebote innerhalb von Ausländerbehörden können zum Beispiel sich unmittelbar an Betroffene wenden und ohne großen Zeit- oder Informationsverlust schwierige Fälle - in anderen Sprachen als Deutsch oder Englisch - begleiten, erklären und kontextualisieren. Über Härtefallkommissionen können vertrackte Fälle in einem klaren Rahmen diskutiert werden und so einen sachlichen, ergebnisorientierten Prozess vorgeben. Meine Daten ergeben zur Wirkung dieser Strukturen ein (noch) eher negatives Bild (vgl. Eule 2014): In vielen Fällen scheint der einzige Vorteil der geschaffenen formellen Kooperationen zu sein, dass informeller Austausch gestärkt wird, während die offiziellen Ziele auf Grund schlechter Verzahnung und unterschiedlicher Interessen noch nicht erreicht werden. Nichtsdestotrotz ist schon der alleinige Versuch, Ausländerbehörde und Zivilgesellschaft in 
eine feste Zusammenarbeitsform zu bringen, bemerkenswert und - zumindest in der Theorie - vielversprechend.

Ich besuche eine Regionalkonferenz der Migrationsberater*innen einer großen zivilgesellschaftlichen Organisation. Ziel ist es, sich über neue rechtliche Veränderungen, besonders schwierige Fälle und ein gemeinsames Vorgehen im Kontext der sich verändernden Flüchtlingszahlen auszutauschen. Im Vorfeld setzen sich verschiedene Vertreter*innen für einen Vormittag mit mir zusammen, um meine Eindrücke des Miteinanders zwischen Ausländerbehörden und zivilgesellschaftlichen Akteuren zu diskutieren. Diese Form von Triangulation meiner Ergebnisse ist häufig besonders nützlich, da hier nicht offene Fragen, sondern vorläufige Analyseergebnisse besprochen werden und die (teils vorher interviewten, teils neuen) Informant*innen so darauf reagieren können.

Meinen Einschätzungen stimmen sie grundsätzlich zu und führen diese weiter aus. Früher habe man gedacht, dass die Ausländerbehörde »der Feind« sei, den es mit allen Mitteln zu bekämpfen gelte. Jetzt könne man viel besser miteinander umgehen, weil man begriffen habe, dass dort nicht nur Idioten und Rassisten säßen. Das Miteinander sei zwar von Behörde zu Behörde unterschiedlich; die Institutionalisierung noch sehr uneben und man sei in vielen Fällen nach wie vor unterschiedlicher Ansicht. Grundsätzlich sei aber im Vergleich zu den Neunzigerjahren das Verhältnis grundsätzlich anders, viel besser, offener, vertrauter. Erfahrene Berater*innen teilen ihre Schauergeschichten, während jüngere Kolleg*innen ihre Erleichterung darüber teilen, dies nicht mehr mitmachen zu müssen.

Die Zunahme ausreisepflichtiger Migrant*innen wird das Verhältnis von zivilgesellschaftlichen Akteuren und Ausländerbehörden stark belasten und wahrscheinlich in vielen Fällen wieder offene Konflikte hervorrufen. Nichtsdestotrotz lohnt es sich, auf die grundlegend unterschiedliche Ausgangssituation gut fünfundzwanzig Jahre früher hinzuweisen. Im Folgenden sollen drei parallel verlaufende Entwicklungen beschrieben werden, die diese Veränderung mitbeeinflusst haben. Erstens hat sich die rechtliche Basis in Deutschland insbesondere in den letzten fünfzehn Jahren grundlegend verändert. Zweitens hat der Ausbau der Integrationsförderung dazu geführt, dass zivilgesellschaftliche Akteure besser ausgestattet, aber auch staatsnaher geworden sind. Drittens haben sich Ausländerbehörden in ihrer Struktur und Ausrichtung stark verändert. 


\section{Ursachen für ein dynamischeres Miteinander}

\subsection{Die Liberalisierung des Migrationsrechts in Deutschland}

Seitdem die sogenannte Flüchtlingskrise eine starke negative öffentliche Reaktion und internen Koalitionsstreit ausgelöst hat, wurde insbesondere das Asylrecht mehrfach verschärft. In diesem Kontext von einer Liberalisierung des Migrationsrechts zu sprechen, mag arg naiv klingen (und die weitere Entwicklung ist nicht abschätzbar), aber der über die letzten fünfzehn Jahre gewachsene Trend ist dennoch nicht zu ignorieren, weil er nach wie vor für völlig andere Rahmenbedingungen sorgt.

Die Geschichte der Revision des Aufenthaltsrechts in Deutschland ist wohlbekannt und an anderen Orten gut nachgezeichnet (vgl. Groß 2004; Geddes 2003; Boswell und Geddes 2011; Bergmann u.a. 2016; Klusmeyer und Papademetriou 2013). Die Revision des Staatsbürgerschaftsgesetzes und die Gegenmobilisierung durch CDU-Politiker alter Schule wie Roland Koch, die Ergebnisse der Süssmuth-Kommission und der erste Entwurf eines neuen Aufenthaltsgesetzes ohne Duldungen, die Blockade desselben im Bundesrat und das 2004 verabschiedete, bis heute hin gültige Aufenthaltsgesetz sind vielfach diskutiert worden. Weniger Aufmerksamkeit bekam die Tatsache, dass sich das Migrationsrecht in Deutschland auch seit 2004 unter den Regierungen Merkel weiterentwickelt hat. Einerseits die Umsetzung von EU-Richtlinien, andererseits Weiterführungen des Bleiberechts für Kinder, der Qualifikationsanerkennung, weiter aber auch länderspezifische Initiativen wie Winterabschiebestopps und die effektive Aussetzung von Abschiebehaft als Zwangsmaßnahme haben Migrationsrecht unter dem Strich weiter liberalisiert. Dieses wurde auch durch Entwicklungen auf europäischer Ebene, wie etwa der Ausweitung der vollen Personenfreizügigkeit auf Rumänien und Bulgarien oder wegweisende EGMR-Entscheidungen im Bereich der gleichgeschlechtlichen Partnerschaft verstärkt. Diese Liberalisierung ist abgesehen von Einzelnormen auch über die zunehmend quantitative Metaerhebung von Rechtsveränderungen ersichtlich (vgl. Helbling 2013; De Haas, Natter und Vezzoli 2014; Huddleston et. al. 2016). Auch mit den aktuellen Verschärfungen des Asylrechts ist die rechtliche Situation für Geflüchtete in Deutschland eine deutlich bessere als zu Beginn der Neunzigerjahre. Dies gilt einerseits für Anerkennungsquoten (und die Tatsache, dass das BAMF 2015 nicht aufgehört hat, Fälle zu bearbeiten), andererseits aber auch für viel bessere Bedingungen für die sprachliche und berufliche Integration und eine (relativ, aber immerhin) humanere und grundrechtlich abgesicherte Abschiebepraxis. Dies wirkt sich natürlich unmittelbar auf die Arbeit von Ausländerbehörden aus. 
Ein erster, wichtiger Grund für das veränderte Verhältnis zwischen Ausländerbehörden und Zivilgesellschaft ist die veränderte rechtliche Grundlage, die den Behörden mehr Spielraum für wohlwollende und positive Entscheidungen gibt. Aufenthaltsrecht ist nicht mehr reine ordnungsrechtliche Gefahrenabwehr (selbst die Einflüsse des Terrorismusbekämpfungsgesetzes sind eher gering, vgl. Eule 2014, Kapitel 4), sondern viel mehr Migrationsmanagement, ein nicht besonders systematischer und klar reformbedürftiger, aber deutlich liberalerer Sortierungsmechanismus als vor 25 Jahren. Dass dies nicht selbstverständlich ist, zeigen tendenziell die Entwicklungen in den vormaligen Hochburgen liberalen Einwanderungsrechts in Europa, den Niederlanden und Großbritannien (vgl. Joppke 2014; Banting und Kymlicka 2012; Michalowski 2011) und strukturell ähnlichen Einwanderungsstaaten mit sehr anderen rechtlichen Dynamiken, insbesondere der Schweiz (vgl. Cattacin und Chimienti 2009; Piñeiro, Bopp und Kreis 2009; D'Amato 2012).

\subsection{Der Ausbau der Förderung von Integration über Sprachkurse und Beratungsangebote}

Neben der rechtlichen Reform wurden in Deutschland in großem Maße Gelder in integrationsfördernde Maßnahmen investiert. Dies betrifft vor allem zwei Bereiche: den Ausbau der Sprachförderung über Deutsch- und Integrationskurse sowie den Ausbau der staatlichen Finanzierung von Migrationsberatung. Dies führte dazu, dass viele zivilgesellschaftliche Akteure ihre Angebote für Migrant*innen ausbauen konnten, aber zur Gewinnung von staatlichen Geldern auch professioneller und weniger konfrontativ werden mussten.

Insbesondere der Bereich der Sprachförderung ist hier hervorzuheben. Auch wenn die Einführung von Sprachkenntnissen als Verschärfung der Einreise- und Aufenthaltsbedingungen verstanden werden kann, zeigt die Praxis, dass die sanktionierenden Elemente wenig bis keine Auswirkung haben (vgl. Michalowski und van Oers 2011; Permoser 2012). Umso beachtlicher ist das finanzielle Engagement, das mit der Sprachpflicht einherging. Obwohl Multikulturalismus in Deutschland für gescheitert erklärt wurde, liegen die staatlichen Ausgaben zur Förderung von Integration und Zusammenleben weit über denen anderer Staaten. Allein das so mit seiner Armut kokettierende Berlin gibt dabei jährlich mehr Geld zur Integrationsförderung aus als der gesamte kanadische Staat (vgl. Collett 2011; Joppke und Eule 2016).

Dieses hat sich unmittelbar auf die Struktur der großen zivilgesellschaftlichen Organisationen (und die kleinerer, lokal agierender) ausgewirkt. Migration ist nicht mehr nur ein politisches Kampfthema, sondern ein wichtiger Aufgaben- 
bereich und Einnahmequelle. Viele Akteure sind nun stärker in staatliche Strukturen eingebettet und schon allein zwecks ihres veränderten Auftrages angehalten, nicht nur konfrontativ mit »Staat" umzugehen. Dieses hat geholfen, die internen Dynamiken in den Organisationen zu verändern und so scheinbar zu einer Form von Professionalisierung und Pragmatismus geführt (vgl. Joppke und Eule 2016).

\subsection{Die Veränderung der Struktur und Ausrichtung von Ausländerbehörden}

Die Feldforschung in Ausländerbehörden und Anschlussinterviews mit weiteren Behörden ergaben, dass die Liberalisierung des Migrationsrechts selbst für die Ausländerbehörden unterschiedliche Auswirkungen hatte. Für einen zunächst kleinen Teil von Ausländerbehörden bedeutete die Reform ein tiefgreifendes Umdenken von Arbeitspraktiken und dem Verhältnis zwischen Zugewanderten und Behörde. Dies waren vornehmlich große Städte mit einer langen Tradition zivilgesellschaftlichen Engagements für Zugewanderte und einfachem Zugang zu starken öffentlichen Medien. Ein zweiter, größerer Teil, veränderte vornehmlich das öffentliche Auftreten der Ausländerbehörde gegenüber Medienvertreter*innen und Zivilgesellschaft, ohne aber an der tatsächlichen Arbeitspraxis viel zu reformieren. Ein dritter Teil schließlich lehnte eine institutionelle Veränderung schlicht ab und versuchte, sich von dem sich wandelnden Einwanderungsdiskurs über weitgehende Abschottung zu schützen. Im Laufe der letzten zehn Jahre hat sich die Gruppe der sich abschottenden Ausländerbehörden allerdings stark verkleinert.

Trotz Vollzugsföderalismus ist dabei auffällig, dass hierbei von Bundesebene Anreize geschaffen werden und leichter Druck ausgeübt wird, um die »zögerlichen« Kommunen zu motivieren, über ihren Abschottungsmodus hinaus zu gehen. In Deutschland wurde etwa 2013 ein zweijähriges Modelprojekt »Ausländerbehörden - Willkommensbehörden« initiiert, welches vom Bundesamt für Migration und Flüchtlinge koordiniert, aber von den Ländern finanziert wird. Hierbei dienen Behörden, die »Willkommenskultur« - also eine Form der Übernahme von liberalen rechtlichen Ideen in Praxis und Arbeitsstruktur - bereits erfolgreich umgesetzt haben, als Vorbilder und profitieren gleichzeitig von dem Austausch mit den Konzepten anderer Behörden (vgl. Eule 2015).

Auch wenn die tatsächlichen Auswirkungen solcher Projekte noch nicht abschätzbar sind - und nicht zu hoch angesetzt werden sollten - ist doch auffällig, dass diese Debatte inzwischen nicht nur öffentlich, sondern vor allem in Praktikerkreisen geführt wird. Auch wenn längst nicht alle der über 400 Ausländerbe- 
hörden »Willkommensbehörden« sind oder sein werden, und auch wenn die Bedeutung von »Willkommensbehörden« in der Regel nicht »Willkommen«, sondern »Auswahl« ist, auch wenn die beforschten Ausländerbehörden eher durch Informalität und Chaos als durch Professionalisierung und Integrationsfreude glänzten, ist eine solche Strukturdebatte nicht grundsätzlich abzutun, denn sie ist neu und hat zumindest bei den »Vorreiterbehörden« enorme Auswirkungen gehabt.

\section{Migrationssteuerung als dynamisches Feld}

Das vorliegende Kapitel hat versucht einen kleinen, aber wichtigen Punkt zu vermitteln: Akteure der Migrationssteuerung und Zivilgesellschaft stehen sich mitnichten grundsätzlich antagonistisch gegenüber, sondern sind zunehmend in nicht reibungsfreien, aber funktionalen und relativ vertrauensvollen Zweckbeziehungen. Ein Blick in die tatsächliche Arbeitspraxis zeigt, dass starre funktionsbezogene Darstellungen nicht ausreichen, um das komplexe und überaus dynamische Miteinander zu verstehen. Auch wenn Ausländerbehörden keine Willkommensbehörden sind, haben sie durchaus vielfach den Willen und die Möglichkeiten, im Rahmen ihres Ermessens (und darüber hinaus) wohlwollend und mit zivilgesellschaftlichen Akteuren gemeinsam Lösungen für Einzelfälle zu finden. Auch wenn zivilgesellschaftliche Organisationen sich primär als Unterstützer von Zugewanderten verstehen, scheinen sie inzwischen so professionalisiert und staatlich eingebunden, dass sie pragmatischen Entscheidungen offen gegenüber stehen und die Grenzen geltenden Rechts als Grenzen ihrer eigenen Mobilisierung für Einzelfälle anerkennen. Auch wenn Vertreter*innen von Zivilgesellschaft und Ausländerbehörden nicht immer einer Meinung sind, können sie sich gegenseitig unterstützen - selbst, wie wir gesehen haben, gegen eigentlich »artverwandte« Stellen und Behörden.

Dies stellt einerseits die Frage, in wie weit zwischen »staatstragenden« und kritischeren zivilgesellschaftlichen Fraktionen unterschieden werden muss - zumindest einige Interviews zu Beginn der Feldforschung scheinen dies zu bestätigen. Viel wichtiger im Kontext der Frage des Verwaltens von Asyl ist aber, wie wir die Ausgangslage verstehen, in der in den kommenden Monaten und Jahren Konflikte um Einzelfälle und Migrationspolitik im Allgemeinen ausgetragen werden. Die Erfahrung der Feldforschung zeigt eindeutig, wie anders die Praxis im Vergleich zur letzten »Flüchtlingskrise« sich verhält. Dies hat mit rechtlichen Veränderungen, finanziellen Investitionen in Integrationsförderung durch Zivilgesellschaft und strukturellen Reformen in Ausländerbehörden zu tun. Bei aller 
berechtigten Kritik an logistischen Verfehlungen, Rechtsverschärfungen, sicheren Herkunftsländern, Abschiebeplänen und vielem Weiteren, sollten die viel positiveren Grundbedingungen, unter denen Geflüchtete unterstützt und in sicherere, integrierende Lösungen gebracht werden können, nicht übersehen werden.

Migrationssoziologisch gesehen kann man das Kapitel schließlich als Ruf für weitere akteursspezifische Forschung verstehen, die der hier vorgestellten Dynamik weitere Nuancen und Datenfülle verleiht. Praxis zu ignorieren oder in Schwarz/Weiß-Verhältnissen zu karikieren, ist nicht nur nach vorliegender Datenlage falsch, sondern auch fatal: Es sind die lokalen Figurationen, in denen zivilgesellschaftliches Engagement am meisten auslösen kann, und die vertrauteren, institutionalisierteren und regen Verbindungen zwischen Zivilgesellschaft und Ausländerbehörden können hier eine wichtige Ressource sein, von der aus politisches, soziales und wissenschaftliches Engagement starten kann.

\section{Literatur}

Anderson, Philip (2011): „Die Wahrung der Menschenrechte von MigrantInnen in der Illegalität auf kommunaler Ebene - das Beispiel München«, in: Maren Mylius/Wiebke Bornschlegl/Andreas Frewer (Hg.), Medizin für Menschen ohne Papiere. Menschenrecht und Ethik in der Praxis des Gesundheitssystems (= Medizin und Menschenrechte, Band 5), Göttingen: V\&R Unipress, S. 173-196.

Banting, Keith/Kymlicka, Will (2012): Is There Really a Backlash Against Multiculturalism Policies?, Stockholm: SULCIS.

Bergmann, Jan/Dienelt, Klaus/Bauer, Ina/Samel, Kai-Christian/Sußmann, Alexandra/Winkelmann, Holger/Wunderle, Simone/Kanein, Werner/u.a. (2016): Ausländerrecht: Aufenthaltsgesetz, Freizügigkeitsgesetz/EU und ARB 1/80, 11. Auflage, München: C.H. Beck.

Borgards, Vera (2006): »Menschen in der Illegalität und die Praxis der Rechtsausübung«, in: Jörg Alt/Michael Bommes (Hg.), Illegalität, Wiesbaden: VS Verlag für Sozialwissenschaften, S. 157-164.

Boswell, Christina/Geddes, Andrew (2011): Migration and mobility in the European Union, Basingstoke: Palgrave Macmillan.

Buckel, Simone (2011): »Urban Governance und irreguläre Migration: Städtische Politik als Handlungsraum im Konfliktfeld irreguläre Migration«, in: Oliver Frey/Florian Koch (Hg.), Die Zukunft der Europäischen Stadt, Wiesbaden: VS Verlag für Sozialwissenschaften, S. 246-262. 
Castles, Stephen (2004a): »The Factors That Make and Unmake Migration Policies«, in: The International Migration Review 38, S. 852-884.

Castles, Stephen (2004b): »Why migration policies fail«, in: Ethnic and Racial Studies 27, S. 205-227.

Cattacin, Sandro/Chimienti, Milena (2009): »Lokale Politik der Eingliederung der Migrationsbevölkerung in der Schweiz - Zwischen Pragmatismus und Populismus«, in: Frank Gesemann/Roland Roth (Hg.), Lokale Integrationspolitik in der Einwanderungsgesellschaft. Migration und Integration als Herausforderung von Kommunen, Wiesbaden: VS Verlag für Sozialwissenschaften, S. 655-672.

Collett, Elizabeth (2011): Immigrant Integration in Europe in a Time of Austerity, Bruxelles: Migration Policy Institute.

Côté-Boucher, Karine/Infantino, Federica/Salter, Mark B. (2014): »Border security as practice: An agenda for research «, in: Security Dialogue 45, S. 195-208.

Cyrus, Norbert/Vogel, Dita (2003): »Work-permit decisions in the German labour administration: an exploration of the implementation process «, in: Journal of ethnic and migration studies 29, S. 225-256.

D’Amato, Gianni (2012): »Switzerland«, in: Christian Joppke/F. Leslie Seidle (Hg.), Immigrant integration in federal countries (= Thematic issues in federalism, Band 2), Montréal: McGill-Queen's University Press, S. 162-191.

De Haas, Hein/Natter, Katharina/Vezzoli, Simona (2014): Growing restrictiveness or changing selection? The nature and evolution of migration policies, Oxford: IMI.

Ellermann, Antje (2009): States Against Migrants: Deportation in Germany and the United States, Cambridge: Cambridge University Press.

Eule, Tobias G. (2014): Inside Immigration Law: Migration Management and Policy Application in Germany, Farnham: Ashgate.

Eule, Tobias G. (2015): »Willkommenskultur auf dem Prüfstand«, in: Christian Joppke/Ben Jann/Axel Franzen (Hg.), Essays on Inequality and Integration. Zürich: Seismo, S. 95-109.

Feldman, Gregory (2012): The migration apparatus: security, labor, and policymaking in the European Union, Stanford: Stanford University Press.

Geddes, Andrew (2003): The Politics of Migration and Immigration in Europe, London: SAGE Publications.

Geiger, Martin/Pécoud, Antoine (Hg.) (2010): The New Politics of Migration Management: Actors, Discourses and Practices, Berlin: Springer.

Geiger, Martin/Pécoud, Antoine (Hg.) (2012): The new politics of international mobility migration management and its discontents, Osnabrück: IMIS. 
Groß, Thomas (2004): »Germany«, in: Imelda Higgins/Kay Hailbronner (Hg.), Migration and asylum law and policy in the European Union, FIDE 2004 national reports, Cambridge: Cambridge University Press, S. 111-134.

Helbling, Marc (2013): »Validating integration and citizenship policy indices«, in: Comparative European Politics 11, S. 555-576.

Hollifield, James (2004): »France: Republicanism and the Limits of Immigration Control«, in: Wayne A. Cornelius (Hg.), Controlling Immigration: A Global Perspective, Stanford: Stanford University Press, S. 157-187.

Huddleston, Thomas/Bilgili, Ozge/Joki, Anne-Linde/Vankova, Zvezda (2016): MIPEX 2015: Germany, Migration Integration Policy Index.

Janis, Irving L. (1972): Victims of Groupthink. A Psychological Study of Foreign-Policy Decisions and Fiascoes, Boston: Houghton, Mifflin.

Joppke, Christian (2014): »European Immigrant Integration after Multiculturalism«, in: Graziano Battistella (Hg.), Global and Asian Perspectives on International Migration, Cham: Springer International Publishing, S. 77-99.

Joppke, Christian/Eule, Tobias G. (2016): „Civic integration in Europe: continuity versus discontinuity«, in: Gary P. Freeman/Nikola Mirilovic (Hg.), Handbook on migration and social policy, Cheltenham: Edward Elgar, S. 342-361.

Klusmeyer, Douglas B./Papademetriou, Demetrios G. (2013): Immigration Policy in the Federal Republic of Germany: Negotiating Membership and Remaking the Nation, Oxford: Berghahn Books.

Michalowski, Ines (2011): »Required to assimilate? The content of citizenship tests in five countries «, in: Citizenship Studies 15, S. 749-768.

Michalowski, Ines/van Oers, Ricky (2011): »How Can We Categorise and Interpret Civic Integration Policies? «, in: Journal of Ethnic and Migration Studies 38, S. 163-171.

Morris, Lydia (2002): Managing Migration: Civic Stratification and Migrants' Rights, London: Routledge.

Permoser, Julia Mourão (2012): „Civic Integration as Symbolic Politics: Insights from Austria«, in: European Journal of Migration and Law 14, S. 173-198.

Piñeiro, Esteban/Bopp, Isabelle/Kreis, Georg (Hg.) (2009): Fördern und Fordern im Fokus. Leerstellen des schweizerischen Integrationsdiskurses, Zürich: Seismo.

Scheffer, Thomas (1995): Aufenthaltsgenehmigung - Studien zur Praxis der Ausländerverwaltung, Bielefeld: Fakultät für Soziologie.

Scheffer, Thomas (2001): Asylgewährung, Stuttgart: Lucius \& Lucius. 